\title{
IRLANDA: REFORMA CONSTITUCIONAL VERSUS CONVENCIÓN CONSTITUCIONAL Análisis del déjà vu de Irlanda desde la perspectiva de la reforma constitucional española
}

\author{
ESTHER SEIJAS VILLADANGOS \\ Profesora Titular de Derecho Constitucional \\ Universidad de León
}

\author{
SUMARIO \\ I. El colapso del tigre celta: la crisis económica \\ en Irlanda. \\ II. La vía de la reforma constitucional \\ III. La vía de la convención constitucional \\ IV. Apuntes desde el constitucionalismo irlandés
}

\section{EL COLAPSO DEL TIGRE CELTA ${ }^{1}$ : LA CRISIS ECONÓMICA EN IRLANDA}

«Todo lo que sube baja» y cuando ese ascenso se produce meteóricamente la caída suele ser estrepitosa. Lejos de un planteamiento científico de la mano de la ley de la gravedad y mucho más cerca de la visión popular de este dicho, Irlanda ha experimentado un comienzo del siglo XXI donde todas las variables a través de las cuales se puede medir el pulso a un Estado han sufrido alteraciones nunca antes experimentadas. En este epígrafe introductorio a un análisis de la reacción constitucional a dicha situación, de la mano de las reformas constitucionales y de la configuración de una Convención Constitucional, trataremos de esbozar los principales rasgos de esa situación convulsa que ha afectado a la

1 La expresión «El Tigre Celta» fue acuñada por el economista británico Kevin Gardiner en 1994. El símil lo adoptó de la locución con que se describían a los tigres asiáticos de la economía: Corea del Sur, Taiwán, Singapur y Hong Kong. GARDINER, K., «The Irish Economy: a Celtic Tiger» MS Euroletter, 31 de agosto de 1994. (http://www.finfacts.com/celtictigereconomyireland.htm). 
totalidad del Estado, pero cuyas manifestaciones en el terreno económico, político, social e institucional han sido especialmente relevantes ${ }^{2}$.

La crisis económica es la enorme y pesada losa que ha marcado la primera década de esta centuria. La variante irlandesa de dicha crisis global ${ }^{3}$ se basó en un modelo de crecimiento insólito en ese pequeño Estado insular. En el año 2000, Irlanda tenía una tasa de inversión extranjera por habitante seis veces superior a la media de la Unión Europea. Empresas de liderazgo mundial tenían su sede en Irlanda (Pfizer, Intel, Ericsson, Google...), circunstancia que la convirtió en el mayor exportador de software del mundo. En 2006, la décima parte de los residentes en Irlanda había nacido fuera. La esencia de este boom la resume Fintan O’Toole muy gráficamente: «simplemente, mucha más gente ubicada en una buena posición para ganar dinero» ${ }^{4}$. Sin embargo, 2001 puede considerarse el año en el que «el tigre celta» empieza a declinar ${ }^{5}$. El detonante fue un cambio de orientación en la política económica del Fianna Fáil, partido dominante en la política irlandesa desde 1932. Si Irlanda había alcanzado la prosperidad porque sus trabajadores generaban productos altamente demandados, el cambio de sistema sería alimentado por otro modelo de crecimiento basado en una constante inflación del precio de los activos financieros, unos bajos tipos de interés, una política de préstamos temeraria y el encumbramiento de la especulación ${ }^{6}$, todo ello unido a una devoción de los irlandeses hacia la propiedad que convertiría a la construcción en el epicentro del modelo económico y que, a la postre, arrastraría a los ciudadanos hacia este tornado. El simbólico hundimiento de un lujoso barco, el Asgard II, financiado con fondos públicos, el 11 de septiembre de 2008 marcó un giró radical en la trayectoria irlandesa. Una revisión de la crisis económica de Irlanda puede periodificarse en torno a tres estadios ${ }^{7}$. Primero, Otoño de 2008. La creación por el gobierno de un salvavidas bancario, llamado NAMA (National Assets Management Agency), un banco de titularidad estatal cuya función principal fue la de absorber los préstamos incobrables hechos por los bancos irlandeses durante el boom. La necesidad de inyectar capital en el Bank of Ireland (Bol) y en el Allied Irish Bank (AIB) en febrero de 2009, junto a la nacionalización del Anglo Irish Bank marcaron la quiebra de las cuentas irlandesas.

2 Sobre esa proyección multifacética de la crisis económica en Irlanda, véase HARDIMAN, N., Irish Governance in crisis, Manchester University Press, Manchester, 2012, págs. 2-16.

3 STIGLITZ, J., «The global crisis, social protection and jobs», International Labour Review, Vol. 148 (2009), n. ${ }^{\circ} 1-2$, págs. 1-2. «La crisis actual afecta a todos los países del mundo... a través de múltiples canales». (http://www.ilo.org/public/english/revue/download/pdf/s1_stiglitz2009_1_2.pdf).

4 O’TOOLE, F., Ship of tools. How stupidity and corruption sank the Celtic Tiger, London, Faber and Faber, 2009, pág. 17.

5 La glosa de esa trayectoria la hallamos en las diversas publicaciones sobre el Tigre Celta. Referencias inexcusables sobre el momento de auge nos remiten a: SWEENEY, P, The Celtic tiger: Ireland's economc miracle explained, Oak Tree Pres, Dublin, 1998. TOOMEY, M., The Celtic tiger: from the outside looking in, Blackhall, 1998. El declive del modelo económico irlandés toma como referencia el trabajo de Peader Kirby, profesor de la Universidad de Limerick. KIRBY, P., The celtic tiger in collapse, Palgrave Macmillan, Basingstoke, 2010.

6 O’TOOLE, F., Ship of tools. How stupidity and corruption sank the Celtic Tiger, op. cit., pág. 21.

7 CHARI, R. y BERNHAGEN, P., «Financial and economic crisis: explaining the sunset over the celtic tiger», Irish Political Studies, Vol 26, n. ${ }^{\circ} 4$, diciembre 2011, pág. 475.

8 Una glosa de los detalles de esta dimensión económica de la crisis puede encontrase en: (http://www.cidob.org/es/documentacion/biografias_lideres_politicos/europa/irlanda/enda_kenny). 
Segundo, Septiembre de 2010. El reconocimiento por parte del Banco Central que las recapitalizaciones bancarias realizadas necesitaban mucho más dinero del previsto inicialmente (45.000 millones de euros), circunstancia que contribuiría a que el déficit público se disparase hasta el $32 \%$ del PIB y la deuda pública hasta el $100 \%{ }^{8}$.

Tercero, Diciembre de 2010. La traslación a la sociedad de esos costes de la mano de recortes en el Estado de bienestar y bajada de las pensiones obligaría a Irlanda a aceptar un paquete de rescate concertado por la Unión Europea y el Fondo Monetario Internacional. Los presupuestos presentados al Dáil, el 7 de diciembre, se enmarcaban en un más amplio plan de austeridad para el período 2011-2014, presidido por la necesidad de cumplir la exigencia de déficit fijado por el Pacto de Estabilidad y Crecimiento de la Unión Europea. El Plan Nacional de Recuperación (NRP) contemplaba reducción del salario mínimo, eliminación de 25.000 puestos laborales en el sector público, recortes en los subsidios y ayudas públicas, ampliación de la base fiscal que suponía la obligación de tributar a parte de los ciudadanos que hasta el momento estaban exentos por ser titulares de rentas bajas y subidas en impuestos directos —el gobierno se resistió a afectar el impuesto de sociedades piedra angular del crecimiento- e indirectos (IVA 23\%).

El sistema político no se mantuvo ajeno a esta crisis. Las elecciones de 25 de febrero de 2011 marcaron un drástico cambio en el liderazgo político aupando al Fine Gael al poder, de la mano de Enda Kenny, quien necesitaría coaligarse con los laboristas ${ }^{9}$. Ese cambio político era sólo la punta del iceberg de la crisis política a gran escala que padecía Irlanda, en cuya esencia hallamos una «crisis de confianza en el sistema político» ${ }^{10}$. Las vías de actuación para afrontar esta crisis política son variadas. Se culpa al sistema electoral (representación proporcional con transferencia de voto $)^{11}$, se plantea la abolición del Senado $^{12}$, se reclama una reducción en el número de parlamentarios ${ }^{13}$, se aboga por una ampliación de los poderes de los Comités parlamentarios, más transparencia en los gastos de los políticos, en suma, la percepción ciudadana de un distanciamiento de la clase

9 GALLAGHER, M., y MARSH, M., (Ed.), How Ireland vote 2011: the full story of Ireland's earthquake election, Palgrave Macmillan, Basingstoke, 2011.

10 HARDIMAN, N., «The impact of the crisis in the Irish political system», Ponencia presentada en el Royal Irish Academy Committee for International Affairs and the global Economic crisis, 5 de noviembre de 2009.

(http://europeandcis.undp. org/uploads/public1/files/Hardiman,\%20\%20The\%20impact\%20of\%20the\%20crisis_\%20RIA,\%205_11_09_\%20FINAL.pdf)

11 Single transferable vote. Un complejo sistema cuya dinámica se puede resumir del modo siguiente. La base es la división en circunscripciones a las que se adjudican determinados escaños. La papeleta de voto incluye todos los nombres de todos los candidatos de todos los partidos de la circunscripción. El elector deber señalar su preferencia con un número de orden $(1,2,3, \ldots)$, a aquellos a quienes quiere dar su voto. Para ser elegido hay que superar un número mínimo de votos, una cuota, que se consigue dividiendo el número de votos válidos emitidos entre el número de escaños más uno. En un primer recuento, el candidato que supera la cuota es elegido, transfiriéndose sus votos sobrantes a quien se hubieran marcado como segunda opción. Por otro lado, si nadie supera esa cuota, entonces se elimina al candidato menos votado y se transfieren sus votos sobrantes al marcado como segunda opción. Esta operación se repite sucesivamente logrando que los candidatos superen la cuota hasta adjudicar la totalidad de escaños de la circunscripción. La regulación constitucional se remite al artículo 16.5. Su desarrollo lo hallamos en la Electoral Act, 1997, reformada en 2011.

12 El cuestionamiento del Senado en un Estado unitario y pequeño, que comparte la lealtad partidista del Dáil ha llevado a la propuesta de su supresión por el Fine Gael. HARDIMAN, N., «Institutional design and Irish political reform», Journal of the Statistical and Social Inquiry Society of Ireland, Vol XXXIX, 2009, pág. 57. 
política de las preocupaciones de la gente corriente. Un auténtico déja vú, para todos aquellos Estados que sufrimos la crisis y, también para los que no, una auténtica constante en la interacción de la política y la sociedad.

En el plano institucional y en la organización del sistema administrativo, un contexto de expansión económica vino a abonar el terreno para la proliferación de agencias públicas, como un recurso alternativo para emplear a más personas sin quebrar aparentemente el número de empleados ministeriales ${ }^{14}$. Igualmente la proliferación de cuerpos consultivos y de asesoramiento ha sido una constante. La concepción de los empleos públicos como un "pago compensatorio» ${ }^{15}$ ha marcado el crecimiento desproporcionado y de imposible financiación del sector público en Irlanda.

El próspero modelo económico del «tigre celta» obvió que su sustento debía engarzarse en la sociedad si buscaba una estabilidad y una perpetuación. De ese modo una de sus grandes debilidades fue el no transferir ese progreso a los ciudadanos ${ }^{16}$. De modo paralelo a la mejora del nivel de vida y a la proliferación de oportunidades de trabajo muy cualificadas, la pobreza y la desigualdad se acrecentó potenciando una importante fractura social en Irlanda. Sobre esa base se erigió un halo de corrupción masiva, determinado por la peligrosa relación simbiótica entre tres actores: el Estado, los banqueros y los constructores-promotores ${ }^{17}$. Paradójicamente, los irlandeses lo soportaron estoicamente, apenas sin protestar, probablemente enmudecidos por el miedo y por la convicción en la ausencia de alternativas ${ }^{18}$.

\section{LA VÍA DE LA REFORMA CONSTITUCIONAL}

Sobre ese contexto esbozado en el epígrafe precedente se han vertebrado dos respuestas, sobre la realidad, la teoría constitucional ha convenido en orientarse de dos modos: la reforma constitucional y la creación de una Convención Constitucional. En este epígrafe trataremos de aproximarnos al marco teórico desde el que se afronta la reforma constitucional en Irlanda, prestando una especial atención al referéndum y vinculando la ilustración del mismo con un estudio de las dos últimas reformas constitucionales que

13 Desde los 166 existentes en la actualidad a 158. Igualmente se postula una reducción en el número de las circunscripciones desde las 43 vigentes a 40. CONSTITUENCY COMMISSION REPORT 2012, Dáil and European Parliament Consituencies, Government Publications, Dublin, 2012, pág. 5. (http://www.constituencycommission.ie/docs/Constit-Rep-2012-Chap-1.pdf).

14 Así lo establece el Informe de la OECD. OECD, Ireland: Towards an integrated public service, OECD, Paris, 2008, págs. 295-298.

15 HARDIMAN, N., «Bringing domestic institutions back into a understanding of Ireland's economic crisis», Irish Studies in International Affairs, Vol XXI, 2010, pág. 87.

16 KIRBY, P., «Lessons from the Irish collapse: taking an international political economy approach», Irish Studies in International Affairs, Vol. 21, 2010, pág. 46.

17 CHARI, R. y BERNHAGEN, P., «Financial and economic crisis: explaining the sunset over the celtic tiger», op. cit., pág. 474.

18 Entrevista a Martin Mansergh, Ministro de Hacienda con el gobierno del Fianna Fail. The Guardian, 30 de septiembre de 2010. (http://www.guardian.co.uk/world/2010/sep/30/ireland-cuts-public-sector-unnemployment). 
han prosperado y con las cuales se ha pretendido afrontar desde la ley suprema la grave situación de crisis que afronta este Estado.

\section{REgULACIÓN DE LA REFORMA CONSTITUCIONAL}

La Constitución de Irlanda (Bunreacht na hÉireann) se aprobó el 1 de julio de 1937, entrando en vigor en diciembre de ese mismo año. Durante un período transitorio hasta junio de 1941, en el que se aprobaron dos reformas a la misma, bastaba una ley ordinaria para afrontar dicho proceso de reforma. Concluido ese tiempo, el referéndum es preceptivo para todo tipo de reforma de la que la Constitución sea objeto. En consecuencia, un total de 23 reformas constitucionales han prosperado en Irlanda ${ }^{19}$. Así, la adhesión a las Comunidades Europeas (1972); la disminución de la edad exigida para votar de 21 a 18 años (1972); la supresión de la posición especial que la Constitución otorgaba a la Iglesia Católica y el reconocimiento a la pluralidad de otras confesiones (1972); adopción (1979); la elección de senadores por determinadas universidades (1979); el derecho a la vida del no nacido en igualdad del derecho a la vida de la madre (1983); la extensión del derecho de voto a determinados ciudadanos no nacionales (1984); Acta Única Europea (1987); Tratado de la Unión Europea (1992); el derecho a la vida del no nacido no limita la libertad para viajar entre Irlanda y otros Estados (1992); el derecho a la vida del no nacido no limita el derecho a acceder a información disponible en otros Estados (1992); divorcio (1995); el rechazo a la petición de fianza por los tribunales a personas que hayan cometido determinados delitos graves (1996); confidencialidad de los debates del gobierno (1997); ratificación del Tratado de Amsterdam (1998); acuerdo entre Irlanda y Gran Bretaña firmado en Belfast el 10 de abril de 1998 (1998); reconocimiento de los gobiernos locales y fijación de las elecciones locales cada cinco años (1999); prohibición de la pena de muerte (2001); ratificar el Tratado regulador de la Corte Penal Internacional (2001); ratificación del Tratado de Niza (2002); concesión de la ciudadanía irlandesa a niños cuyos progenitores no fuesen nacionales (2004); ratificación del Tratado de Lisboa (2009); reducción del sueldo de los jueces (2011) y, por último, la trigésima reforma relativa a la ratificación del Tratado sobre Estabilidad, Coordinación y Gobernanza en la Unión Económica y Monetaria (2012).

La Constitución irlandesa puede ser objeto de reforma en su totalidad, lo que por ejemplo ha llevado a sostener que el poder de reforma es ilimitado ${ }^{20}$, no obstante este proceso no puede circunscribirse a una actuación exclusiva del legislativo, sino que se requiere un referéndum, por lo que el calificativo de «rígida» es, igualmente, apropiado.

Los principales rasgos que ayudan a comprender la reforma constitucional de Irlanda pueden desglosarse del siguiente modo.

19 Es importante tener en cuenta que en la ordenación de las enmiendas se computa también aquellas que no han prosperado por no superar el preceptivo referéndum o estancarse en la previa tramitación parlamentaria. Así, no hay una reforma $12 .^{\circ}$ relativa al derecho a la vida de los no nacidos, rechazada en referéndum el 25 de noviembre de 1992; la $22^{\circ}$, relativa a la remoción de jueces, no siendo aprobada la respectiva ley en el parlamento en 2001; la 24a , relativa al Tratado de Niza, rechazada el 7 de junio de 2001 y la $25^{\circ}$ relativa a la protección de la vida humana en el embarazo, propuesta rechazada el 6 de marzo de 2002.

20 CASEY, J., Constitutional Law in Ireland, Round Hall Sweet \& Maxwell, Dublín, 2000 (3. a edic.), pág. 709. 


\section{a. Aproximación formal: el proceso de reforma.}

La regulación de la reforma constitucional se desarrolla en el art. 46 de la Constitución $^{21}$. La iniciación de un proceso de reforma se remite a la presentación de un proyecto de ley en la Cámara Baja del Parlamento, desde el que tras su tramitación se remitirá al Senado. Una vez aprobada en ambas cámaras se someterá a referéndum. La denominación que ha de presidir ese proyecto de ley ha de incorporar expresamente su designación como de reforma de la Constitución. Una vez cumplidos esos trámites el Presidente de la República de Irlanda lo firmará, previa verificación del cumplimiento de estos requisitos procesales, y procederá a su promulgación entrando en vigor.

\section{b. Aproximación material: el contenido de la reforma}

El contenido de la reforma es el más amplio que se puede concebir, ya sea modificando, agregando o derogando uno o todos los preceptos de la Constitución. Incluso, en esa facultad de reforma se incluye el poder de «clarificar o hacer más explícito algo ya regulado en la Constitución ${ }^{22}$. Existe una libertad de los ciudadanos, canalizada a través del sistema de representación parlamentaria para variar el sentido de la Constitución en la dirección que ellos consideren. Así, estaríamos ante una ausencia de límites a la soberanía popular ${ }^{23}$.

La materialización de estas reformas puede reflejarse en un texto anexo, es decir la incorporación de un documento extraño al articulado de la Constitución. Es el caso de la vigesimoquinta enmienda, que no prosperaría, relativo a la protección de la vida humana durante el embarazo que remitía a una ley sobre esa cuestión de $2002^{24}$. Igualmente cabe postular un supuesto de una reforma calificable de multifacética, por cuanto podría afectar a diferentes preceptos de la Constitución. Es el caso de las enmiendas $19^{\circ}$ y $21^{\circ}$, referidas al Acuerdo entre Gran Bretaña e Irlanda y a la prohibición de la pena de muerte, respectivamente. Por el contrario, no se permite una reforma indirecta de la Constitución, contrario sensu, las reformas han de ser expresas y precisas en su contenido.

21 Art. 46 Constitución de Irlanda: «1. Todo precepto de la presente Constitución puede ser reformado por vía de modificación, adición o derogación, en la forma prevista en este artículo.

2. Toda propuesta de reforma de esta Constitución será iniciada en la Cámara de Representantes como proyecto de ley, y una vez haya sido aprobada por ambas Cámaras del Parlamento, será sometida a referéndum popular de acuerdo con la ley sobre referéndum en vigor en ese momento.

3. Todo proyecto de ley de esta naturaleza llevará el nombre de «Ley de reforma de la Constitución».

4. Un proyecto de ley que contenga una o más propuestas de reforma de esta Constitución no contendrá ninguna otra propuesta.

5. Un proyecto de ley que contenga una propuesta de reforma de esta Constitución será firmado por el Presidente de la República, una vez que éste tenga la certeza de que las disposiciones de este artículo han sido cumplidas y de que dicha propuesta ha sido debidamente aprobada por el pueblo, de acuerdo con las disposiciones de la sección 1 del artículo 47 de esta Constitución, será debidamente promulgada por el Presidente de la República como ley».

22 KELLY, J.M., The Irish Constitution, Lexis Nexis, Dublín, 2003, pág. 2092.

23 En una trilogía de casos en los 90, el Tribunal Supremo afirmó la supremacía de la soberanía popular expresada a través del referéndum como principio clave de la Constitución. El tribunal llegó a calificar de «sacrosanta» a la misma. KELLY, Ibídem, pág. 2097.

24 «Protection of Human Life in Pregnancy Act 2002» que debería ser aprobada en los 180 días posteriores a la aprobación de la reforma. 


\section{c. Judicialización de la reforma de la Constitución}

El proceso de judicial review ha encontrado un serio obstáculo en los procesos de reforma constitucional. Así, no existe una potestad judicial para revisar una ley que haya reformado la Constitución, lo cual no obstante parece no haber constituido un impedimento para que los ciudadanos litiguen buscando la anulación judicial de diversas reformas constitucionales. El principio de separación de poderes lleva a prevenir al poder judicial de invadir el espacio correspondiente al poder legislativo. El único reducto que comprendemos viable para tal revisión es el de las potenciales irregularidades procesales, ej. Una propuesta de reforma que no se tramitase convenientemente en el parlamento, o que no lo hiciese con la expresa denominación de «ley de reforma de la Constitución», como requiere el art. 46.3 , sí podría ser abrogada por un tribunal.

\section{d. Impulsos a la reforma constitucional}

Los procesos de reforma constitucional han sido motivados en Irlanda, en 1966 y en 1996, por sendos Comités sobre la Constitución. Estamos ante una recomendación oficial sobre las reformas a realizar. En 1966 el Comité de todos los partidos del Parlamento realizó un informe que culminó con la reforma de la Constitución en lo concerniente al papel de la Iglesia Católica (5.a enmienda). A partir de ahí el trabajo de este Comité se diluyó, procediendo a su desaparición en 1968. Treinta años después, se procedió a crear el Grupo para la revisión de la Constitución ${ }^{25}$, que procedió a revisar íntegramente la misma, formulando numerosas sugerencias para el cambio. Este informe constituyó la base para el trabajo del Comité de todos los partidos del Parlamento ${ }^{26}$ y se ha plasmado en diferentes informes publicados desde 1997. Así, sobre la reforma del Senado, del Presidente, de los Tribunales y del poder judicial, del referéndum o del parlamento. De todas ellas, sólo tres se han materializado en reformas efectivas de la Constitución, en materia de confidencialidad de los debates del gobierno, gobiernos locales y abolición de la pena de muerte, aspectos de menor calado del conjunto de las diferentes propuestas formuladas.

\section{EL REFERÉNDUM COMO INSTRUMENTO CLAVE EN LA REFORMA CONSTITUCIONAL}

\section{a. Marco normativo}

La regulación referida al referéndum se contiene en los artículos 27,46 , pero especialmente en el artículo $47^{27}$ de la Constitución de Irlanda de 1937. La Ley electoral de 1992 (the Electoral Act) y sus respectivas reformas de 1996, 1997, 2001, 2004, 2006. La

25 http://politicalreform.ie/2011/06/28/the-constitution-review-group-report-a-great-free-resource-forirish-citizens/

26 http://www.oireachtas.ie/viewdoc.asp?fn=/documents/Committees29thDail/constitution.htm.

27 Art. 47 «1. Toda propuesta de reforma de esta Constitución que sea sometida por referéndum a decisión popular, a los efectos del artículo 46 de esta Constitución, se considerará aprobada por el pueblo, si la mayoría de los votos de dicho referéndum han sido favorables a que su conversión en ley. 
Ley reguladora del Referéndum de 1994, reformada en 1998 y en 2001 y la Ley reguladora de los Ministerios y Secretarías, en su reforma de $2001^{28}$.

\section{b. Aproximación procesal}

El referéndum es un elemento clave en el proceso articulado constitucionalmente para reformar la Constitución (art. 46). Una vez concluida la tramitación parlamentaria, el texto de la propuesta de reforma constitucional debe ser remitida a aprobación plebiscitaria de los ciudadanos mediante referéndum.

En el referéndum puede votar todo ciudadano de Irlanda, con residencia habitual en el Estado, mayor de 18 años y que conste como tal en un Registro de electores, común a las elecciones legislativas. Una vez concluida la tramitación parlamentaria de una propuesta de reforma constitucional, el Ministro de Medio Ambiente, Comunidad y Gobierno Local designa un responsable para la gestión del referéndum (Referendum Returning Officer) quien dirigirá el proceso del referéndum, incluyendo el recuento y la proclamación oficial de resultados. Una Orden del Ministro de Medio ambiente, Comunidad y Gobierno Local determinará la fecha del referéndum, en un plazo entre 30 y 90 días posteriores a esa orden de convocatoria.

En el proceso de desarrollo del referéndum la Comisión para el referéndum juega un importante papel, como veremos de inmediato.

El carácter del voto es secreto, puede emitirse personalmente o por correo.

El contenido de la papeleta de voto muestra el título del Proyecto de Ley de reforma de la Constitución. El votante ha de indicar si aprueba o rechaza dicha propuesta, marcando con una X la casilla del sí o del no en la papeleta. El recuento de los votos se produce en las circunscripciones. Tras la publicación provisional de los resultados se abre un período de 7 días para su posible impugnación ante el Tribunal Superior de Justicia (High Court). Transcurrido ese período sin haberse interpuesto ninguna demanda los resultados provisionales devienen en finales y si se ha producido el triunfo del si, el proyecto de ley es firmado por el Presidente, procediéndose así a la reforma de la Constitución.

\section{c. Judicialización del referéndum}

Existe una constante en los diferentes refenda, conexa a la naturaleza de los mismos, de abordar cuestiones políticas explosivas ${ }^{29}$. La consecuencia de ello es el recurso a la judicialización, tanto del proceso, como de los resultados del mismo.

2.1 $1^{\circ}$ Toda propuesta que, no siendo una propuesta de reforma de la Constitución, sea sometida por vía de referéndum a decisión popular, se considerará rechazada por el pueblo si la mayoría de los votos de dicho referéndum se pronuncia en contra de su promulgación como ley y si esta mayoría suma no menos del treinta y tres coma treinta y tres por ciento de los votantes inscritos en el censo.

$2^{\circ}$ Toda propuesta que, no siendo una propuesta de reforma de la Constitución, se someta por vía de referéndum a decisión popular, se considerará aprobada por el pueblo, conforme al artículo 27 anterior, a menos que fuera rechazada por los votantes de acuerdo con las disposiciones de la subsección anterior de este artículo.

3. Todo ciudadano que tenga derecho a votar en las elecciones a miembros de la Cámara de Representantes tendrá derecho a votar en un referéndum.

4. Con observancia de lo dispuesto anteriormente, el referéndum se regulará por una ley».

28 Department of the Environment, Community and Local Government, The Referendum in Ireland, Department of the Environment, Dublin, 2012, pág. 10.

29 BAIRBRE, O., «The referendum process in Ireland», Irish Jurist, n. ${ }^{\circ}$ 35, 2000, pág. 305. 
El enjuiciamiento de los términos en que se ha formulado la consulta del referéndum ha sido objeto de revisión judicial, pudiéndose resumir la posición judicial en que ante «una duda sobre la claridad de la formulación, el ciudadano tiene la opción de votar en contra», por lo que el cuestionamiento en la formulación de la pregunta no afectaría a la validez del mismo ${ }^{30}$.

Una de las cuestiones más controvertidas ha sido dilucidar el papel que el gobierno ha de jugar en un referéndum. La cuestión de fondo es si el gobierno debe propiciar una campaña imparcial en la que difunda publicidad sobre ambas opciones a votar o si por el contrario está legitimado a inclinarse por una de ellas. La cuestión se llevó a los tribunales en el referéndum de ratificación del Tratado de Maastricht. El referente es el caso McKenna vs An Taoiseach, que tras la apelación al Tribunal Supremo llevaría a concluir «la impropiedad constitucional» atribuible al gobierno cuando gasta fondos públicos en una campaña dirigida a influir en los votantes en favor de la reforma propuesta y no en abogar o hacer campaña por la convocatoria del referéndum en $s^{31}$. Este pronunciamiento marcaría que el Grupo de Revisión de la Constitución recomendase la provisión de manera equitativa de la asignación de fondos asignados para la celebración de un referéndum, esta recomendación derivó en la creación de una Comisión independiente del referéndum en la Ley del Referéndum de $1998^{32}$.

Finalmente, la impugnación de los resultados del referéndum ha de canalizarse al Tribunal Superior de Justicia, previo pago de una tasa por los demandantes de $5000 €$, que para derivar en la anulación del mismo será preciso que se pruebe que las irregularidades cometidas (influencias indebidas, sobornos, ruptura del secreto, impedimentos en la celebración del referéndum, fallos procesales) hayan afectado prima facie a los resultados.

\section{LAS REFORMAS de la CONSTITUCión DE IRLANDA COMO RESPUESTA A LA CRISIS ECONÓMICA}

La Constitución ha sido una herramienta con la que afrontar la crisis económica. Sobre la idoneidad de la misma se ha debatido mucho recientemente ${ }^{33}$. Marcados por los fines acotados de este trabajo podemos sostener que la reforma constitucional es un elemento coadyuvante a las diferentes medidas que se pueden adoptar para afrontar un proceso de severa recesión. La prioridad son las medidas económicas y fiscales, la reforma

30 Caso Roche vs. Ireland, 17 de junio de 1983 HC, Cfr. DOYLE, O., Constitutional Law, Text, Cases and Materials, Clarns Press, Dublín, 2008, pág. 405.

31 KELLY, J.M., The Irish Constitution, op. cit., pág. 2014.

32 El papel fundamental de esta Comisión es explicar al electorado el objeto del referéndum. Para ello procederá a emitir una serie de manifiestos en los que transmitirá dicha explicación, así como las informaciones que estime relevantes para dicha convocatoria. Igualmente es de su competencia dar publicidad y distribuir esos comunicados por todos los medios, incluyendo el uso de la televisión, la radio y otros medios electrónicos en la medida en que la Comisión los considere más idóneos para captar la atención del electorado y para asegurar en todo lo posible el acceso a esa información a personas con discapacidades auditivas o visuales. Finalmente, la reforma de 2001 de la Ley reguladora del referéndum establece en su art. 1.c, (Referendum Act 2001, 22 de diciembre) que será función de la Comisión «promover una concienciación pública con el referéndum y estimular al electorado a votar en el mismo.».

33 EMBID IRUJO, A., La Constitucionalización de la crisis económica, Iustel, Madrid, 2012, págs. 35-47. 
de la Constitución supone una reafirmación normativa de las mismas en el máximo grado en que ésta puede emitirse, acorde al carácter de ley suprema de la Constitución, cuya virtualidad es esencialmente metanormativa. Esa trascendencia jurídica se proyecta en dos frentes, ad intra, reforzando el acuerdo entre las diversas fuerzas políticas, que se evidencia inexistente en el trabajo legislativo ordinario, recabando la atención de todos los ciudadanos sobre la relevancia de las medidas adoptadas y apelando a su compromiso con la consecución de las diversas medidas que traten de implementarla. Ad extra, enviando un mensaje de meridiana claridad a todos aquellos interlocutores y agentes activos tanto en generar la crisis como en contribuir a paliarla, marcados por esas coordenadas globales que están presentes en la misma pero que no eluden las prevalentes responsabilidades internas.

Así, desde Irlanda el contexto de crisis ha avocado a apelar, desde una reforma total de la Constitución, abrazando una nueva Constitución para una Segunda República ${ }^{34}$, hasta un catálogo abierto de reformas concretas, de la mano de una Convención Constitucional que analizaremos de inmediato. El punto de referencia es el programa de gobierno postulado por la coalición Fine Gael-Laboristas ${ }^{35}$, vencedora en las elecciones de marzo de $2011^{36}$.

Cinco prioridades fueron fijadas en dicho programa de gobierno para afrontar la reforma constitucional:

- Abolición del Senado.

- Ampliar la capacidad de actuación de los Comités del Parlamento (Oireachtas) a los efectos de llevar a cabo investigaciones completas sobre diferentes materias, limitados por decisión jurisprudencial ${ }^{37}$.

- Realizar un referéndum para proteger los derechos de los ciudadanos a comunicarse confidencialmente con los representantes políticos.

- Convocar un referéndum para reformar la Constitución para permitir que el Estado reduzca los sueldos de los jueces en determinadas circunstancias como parte de un recorte general de los gastos del sector público.

- Convocar un referéndum para reformar la Constitución a los efectos de potenciar que los derechos de los niños sean respetados, siguiendo las líneas recomendadas por el Comité interpartidos del Parlamento (All-Party Oireachtas Committe).

34 O'TOOLE, F., Ship of tools. How stupidity and corruption sank the Celtic Tiger, op. cit., págs. 212-224; O'SULLIVAN, M., «We need a political system that encourages strategic thinking», Irish Times, 14 de abril de 2009.

35 Programme for Government 2011 (6 de marzo de 2011), pág. 17.

36 Los resultados de las elecciones pueden consultarse en: (http://electionsireland.org/results/referendum/refresult.cfm?ref=2012R). Una publicación de referencia es: DONNELLY, S., Elections 2011, Donelly, Dublín, 2012, 560 págs.

37 El caso Maguire versus Ardagh, conocido como el caso Abbeylara. Un subcomité del Parlamento inició una investigación sobre un suceso ocurrido en Tonnymore, Abbeylara, en el que falleció un hombre de un disparo. Para ello se instó la comparecencia de los acusados. El Tribunal Supremo resolvió que los Comité del parlamento no tiene poder para iniciar procesos que exijan la comparecencia de personas que no sean miembros del parlamento, presten declaraciones y realicen aportaciones documentales. Se considera una actuación ultra vires de los poderes de las Cámaras. (http://www.courts.ie/__80256F2B00356A6B.nsf/0/ 0C0D2AA3FE4472C880256CAA003D13AA?Open\&Highlight=0,Ardagh, language_en ). 
Hasta la fecha sólo una de esas propuestas, conectada directamente al contexto de crisis económica, se ha transformado en una reforma efectiva de la Constitución: la enmienda vigesimonovena referida a la remuneración de los jueces. Está pendiente para el otoño de 2012 la celebración del referéndum en materia de potenciación de los derechos de los niños en la Constitución. La agregación de una trigésima enmienda, de la que se celebraría el referéndum el 31 de mayo de 2012, referida a la ratificación por Irlanda del Tratado de Estabilidad, Coordinación y Governanza de la Unión Económica y Monetaria, no avanzada en ese catálogo, nos ofrece un interesante marco en el que evidenciar esa conexión crisis-Constitución que perseguimos en esas líneas.

La que se convertiría en la vigésimo novena reforma de la Constitución de Irlanda, merced a la Ley «Twenty_Ninth Amendement of the Constitution (Judges' Remuneration) Act $2011 »$, de 17 de noviembre de ese año, procedió a suplir el rotundo apartado quinto del artículo 35, «La remuneración de un juez no podrá ser reducida durante su permanencia en el cargo» por otra redacción más acorde con los principios de igualdad, especialmente igualdad en el esfuerzo para superar una crisis económica. Así, el texto de la reforma es el siguiente (art. 35.5 ): «1. ${ }^{\circ}$ La remuneración de un juez no podrá ser reducida durante su permanencia en el cargo, salvo lo establecido en este artículo. $2{ }^{\circ}$ La remuneración de un juez está sujeta al pago de impuestos, tasas u otros gravámenes que se apliquen legalmente a todas las personas o aquellas que pertenezcan a un grupo en particular. $3 .^{\circ}$ Donde, antes o después de la aprobación de este artículo, se haya hecho o se haga una reducción de las retribuciones, fijada legalmente, a las personas pagadas a cargo del erario público y en esa ley se establezca que dichas reducciones son por interés público, se adoptará una disposición legal similar a los efectos de aplicar proporcionalmente dichas reducciones a la remuneración de los jueces».

La tramitación del proyecto de Ley, conforme a las exigencias del art. 46 de la Constitución se inició el 2 de agosto de 2011 en la Cámara Baja. Su tramitación fue rápida $^{38}$, con un gran consenso debido fundamentalmente a que se trataba de aplicar medidas a los jueces propuestas por el anterior gobierno para todos los funcionarios, concluyéndose en el Senado el 21 de septiembre de $2011^{39}$. El referéndum se celebraría el 27 de octubre de 2011, obteniendo el sí un voto favorable del 79,79\% de los votos. La firma presidencial, el 17 de noviembre de 2011, formalizaría y cerraría este proceso de reforma constitucional. Del contenido material de la reforma hemos de destacar el énfasis puesto en todos los debates sobre la necesidad de compatibilizar esta reforma con un respeto estricto a la independencia judicial y la prioridad de utilizar todos los medios posibles para luchar contra la crisis, reduciendo el déficit. Se trata en esencia de aplicar la Financial Measures in the Public Interest Act, 2009 a los jueces. Básicamente se trata de aplicarles una reducción de media del $15 \%$ de su sueldo, una moratoria para quienes opten por la jubilación antes del 29 de febrero de 2012 de no considerar esa reducción para el cómputo de la pensión y de aumentar las cantidades abonadas por los jueces a las ar-

38 La complejidad de la redacción, especialmente el tercer párrafo, unida a esa tramitación express en agosto, suscitó críticas entre los ciudadanos y la doctrina. FELDMAN, E., «The referendum judicial pay is illconceived», Irish Times, 16 de septiembre de 2011.

39 Seanad Éireann Debate, vol 210, n. ${ }^{\circ}$, págs. 221-223. http://debates.oireachtas.ie/sea$\operatorname{nad} / 2011 / 09 / 21 / 00010$.asp 
cas del Estado para el pago de las cuotas de la seguridad social. El ahorro estimado se calculaba por el Ministro de Justicia en dicho debate en torno a los 5,5 millones de euros. Con estos datos, no podemos dejar de calificar esta enmienda como un elemento simbólico en la lucha contra el excesivo déficit del Estado irlandés, al extender a los jueces los sacrificios exigidos a los otros trabajadores públicos y a todos los ciudadanos. La legitimidad la hallamos en la aplicación material del principio de igualdad, eliminando cualquier reducto de privilegios blindado a los sacrificios que exige la lucha contra la crisis.

La reforma constitucional que más directamente ha afrontado la crisis económica en Irlanda es la referida a la trigésima enmienda, referida a la ratificación del Tratado de Estabilidad, Coordinación y Gobernanza de la Unión Económica y Monetaria, aprobado el 2 de marzo de 2012. El contenido de la enmienda destinada a reformar el art. 29, agregando este epígrafe en el apartado $10^{\circ}$ a su dilatada redacción, es el siguiente: «El Estado puede ratificar el Tratado de Estabilidad, Coordinación y Gobernanza de la Unión Económica y Monetaria, hecho en Bruselas el 2 de marzo de 2012. Ninguna disposición de la presente Constitución deroga las leyes aprobadas, actos realizados o medidas adoptadas por el Estado, que sean necesarias como consecuencia de las obligaciones adquiridas por el Estado en el marco de ese Tratado, ni impide que las leyes aprobadas, actos realizados o medidas adoptadas por los órganos competentes en el marco de ese Tratado tengan vigencia en el Estado».

El iter de esta reforma comienza en su presentación en el Dáil el 28 de marzo de 2012. Su tramitación traduce el acuerdo de los dos partidos más relevantes, el Fine Gael y el Fianna Fail, que lideraran dicha tramitación y el voto favorable en el ulterior referéndum que se convocaría para el día 31de mayo de 2012. Irlanda, que rechazaría en 2001 el Tratado de Niza y en 2008 el de Lisboa, no parecía el terreno mejor abonado para someter este Tratado a referéndum. Empero, el contenido del Tratado no deja lugar a dudas, junto al límite al déficit público y las sanciones aparejadas casi automáticamente a quienes superen el umbral del 3\% de déficit, el Tratado prevé que aquellos Estados que lo rechacen no tendrán acceso a las ayudas del Fondo de rescate, del que Irlanda hasta la fecha había recibido 85.000 millones de euros. Con estos condicionantes el voto favorable en el referéndum de un 60, $29 \%$ no ha de extrañar. La misma lectura la tiene la elevada cota de abstención, aproximadamente el 50,6\%, de los más de 3 millones de electores inscritos para votar. El margen para el no era prácticamente inexistente. La activa campaña del gobierno ${ }^{40}$ contrarrestó la tímida resistencia al Tratado liderada por el Sinn Fein. El proceso formal de reforma constitucional ha culminado el 28 de junio de 2012, con la firma por el Presidente Michael D. Higgings de la Ley de reforma. Dos palabras marcaron el proceso, la necesaria confianza que su inserción constitucional iba a generar y su carácter decisivo para la recuperación. La reforma constitucional de Irlanda en aras a afrontar la crisis se concibe así como un paso decisivo, no el único, ni el último, pero exterioriza un blindaje del compromiso para reconducir la situación económica. El principal interrogante deriva de la desconexión de los ciudadanos a esta reforma. Ese nexo social podría paliarse de la mano de la Convención Constitucional. En las siguientes líneas profundizaremos en la misma.

40 http://www.stabilitytreaty.ie/

UNED. Teoría y Realidad Constitucional, núm. 30, 2012, pp. 411-432. 


\section{LA VÍA DE LA CONVENCIÓN CONSTITUCIONAL}

\section{Concepto y naturaleza de la Convención Constitucional}

Articular un marco teórico sobre la naturaleza de la Convención Constitucional es una tarea que trasciende la finalidad de este trabajo, pero a la par se presenta como una referencia ancilar e inexcusable si queremos otorgarle un mínimo empaque. Con esa premisa procedemos a esbozar una exigua referencia doctrinal de la Convención Constitucional, fundamentalmente a partir de los trabajos de Dicey, Jennings, Wheare y Bagehot. La conclusión que perseguimos es buscar un enlace de esa teoría con el supuesto concreto de Convención Constitucional planteado en Irlanda como instrumento articulado para avanzar en la reforma de la Constitución.

Afirma Dicey que «las Convenciones de la Constitución consisten en costumbres, prácticas, máximas o preceptos que no están aplicados o reconocidos por los tribunales y que constituyen un conjunto no de leyes, sino de principios constitucionales o políticas» ${ }^{41}$. La Convención Constitucional es así un instrumento para flexibilizar la Constitución, para «cubrir con un manto de carne los secos huesos de la ley» ${ }^{42}$.

Es decir, la esencia de la Convención Constitucional es que carece de un basamento legal, especialmente constitucional, al que por otro lado se vincula y al que busca adaptar a la práctica política para conseguir, de algún modo, lo que Loewenstein llamaba Constitución normativa, esa comunión entre la literalidad de la Constitución y la implementación de la misma ${ }^{43}$.

Una vez definida la Convención Constitucional hay dos aspectos especialmente relevantes en su cuerpo dogmático: el origen y la fuerza obligatoria de las mismas. En lo que respecta al origen de la Convención Constitucional podríamos reconducir a tres las raíces de ésta. Primero, una conducta que ha persistido a lo largo de un gran y dilatado período de tiempo y que en un primer estadio tenía un carácter persuasivo, para luego convertirse en obligatorio ${ }^{44}$. Segundo, un acuerdo para trabajar de una forma particular y adoptar una pauta singular de conducta. Así concebida, esta Convención tendría un carácter normativo vinculante de modo inmediato. Su eficacia proviene no de un pasado histórico como uso, sino de la fortaleza del acuerdo ${ }^{45}$. Tercero, lo que podríamos designar como razonabilidad constitucional. Una convención puede surgir sobre la base de un re-

41 DICEY, A., The study of the law of the Constitution, Macmillan, London, 1964, pág. 417.

42 JENNINGS, I., The law and the Constitution, London University Press Ltd, London, 1967, (5 edic.: 6. ${ }^{\mathrm{a}}$

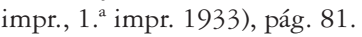

43 LOEWENSTEIN, K., Teoría de la Constitución, Ariel, Barcelona, 1970.

44 McWHINNEY, E.M., «Constitutional Conventions», en Canadian Bar Review, vol. XXV, 1957, págs. 92-96. Se analiza la práctica ejecutada por la Reina Isabel II de Inglaterra de realizar diversas consultas tras la dimisión del Primer Ministro Británico, Sir Anthony Eden (al propio ministro dimisionario, a Sir Winston Churchill, en condición de único ex-Primer Ministro conservador vivo y al Marqués de Salisbury, uno de los miembros más influyentes del partido conservador) en enero de 1957. Sobre la base de un consejo unánime la Reina propuso al Ministro de Economía, Harold Macmillan, prescindiendo de otro candidato más experimentado R. A Butler. En esa práctica, descrita como Convención Constitucional, se apoyaba en otra actuación semejante de su abuelo Jorge V, tras la dimisión del Primer Ministro Bonar Law en 1923.

45 WHEARE, K., Modern Constitutions, Oxford University Press, London, 1975 (3. ${ }^{\circ}$ impr., 1..$^{\circ} 1960$ ), pág. 122. 
conocido principio de gobierno, que se avala por una razón o justificación para ello ${ }^{46}$. Son, precisamente, estos dos últimos los que se aplican al caso de la Convención Constitucional de Irlanda, cuya finalidad se acomoda perfectamente a la que la doctrina atribuye a las Convenciones Constitucionales domésticas, la de dar efecto a los principios de responsabilidad gubernamental ${ }^{47}$.

Sentada la propedéutica que antecede y, en ejercicio de una metodología deductiva, la aplicación al caso concreto de la Convención Constitucional Irlandesa de dicha teoría nos lleva a describirla del siguiente modo. «Asamblea, reunión o agrupación de ciudadanos y sus representantes, avalados por precedentes organizativos similares y por el compromiso político de gobernabilidad basado en el poder discrecional del ejecutivo, con la finalidad de dotar de un suplemento al contenido normativo de la Constitución, concretamente en lo referido a la reforma constitucional, a los efectos de potenciar su flexibilidad, adoptándolas a las circunstancias cambiantes de la vida nacional, a unas nuevas necesidades, determinadas en este caso por una coyuntura de crisis». Con ella se reforzaría esa parte eficiente, presente en cada Constitución ${ }^{48}$.

\section{Otras eXPerienCias de CONVEnCión CONSTitucional}

El término de Convención constitucional lleva implícito una gran resonancia histórica que nos lleva a visualizar los personajes de Thomas Jefferson o James Madison en la Convención de Filadelfia de 1787, en lo que sería el asentamiento de las bases del constitucionalismo moderno ${ }^{49}$. Empero, un análisis retrospectivo más inmediato nos insta a recuperar unos concretos ejemplos más cercanos del recurso a las convenciones constitucionales como elemento reactivador de los cambios constitucionales en su acepción más lata. Australia, años 90, y el planteamiento de un cambio pro republicano de la forma de gobierno; la Unión Europea y la redacción de la Carta de Derechos Fundamentales de la Unión, años 1999-2000 o las provincias canadienses de Columbia Británica y Ontario, en 2003-2004 y 2006, respectivamente, en relación a reformas en sus sistemas electorales son los tres supuestos en los que la opción de la Convención Constitucional se ha convertido en un recurso de naturaleza similar al que actualmente se diseña en Irlanda y sobre los cuales se ha inspirado ${ }^{50}$.

Uno de los referentes, especialmente relevante en lo atinente al caso irlandés ${ }^{51}$, del uso de la Convención constitucional es el debate acaecido en Australia en los años 90 acerca de la transformación del Estado australiano en una república y, en consecuencia, en la sustitución del monarca, como jefe de Estado, por un presidente. El proceso desarro-

46 MARSHALL, G., Constitutional Conventions. The rules and forms of political Accountability, Clarendon Press, Oxford, 1984, pág. 9.

47 Ibídem, pág. 18.

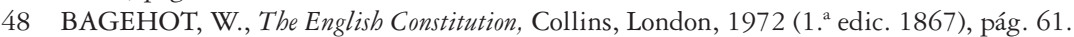

49 O’MAHONEY, C., «This so-called Constitutional Convention is a charade», Irish Times, 7 de junio de 2012.

50 ODOWD, T. J., «The sovereign debt crisis and the new boundaries of the Irish State», Comunicación presentada en la Conferencia de EPLO, Malta, 8 y 9 de Julio de 2011, (http://papers.ssrn.com/sol3/papers.cfm?abstract_id=1946323) pág. 8. 
llado puede estructurarse en tres estadios: primero, las deliberaciones y el informe del Comité Asesor sobre la República (Republic Advisory Committe) en 1993; segundo y más importante a los efectos que nos ocupan, la organización de una Convención constitucional en 1998 y, el punto final, referido a la celebración de un referéndum en 1999 en el que, por cierto, dicha propuesta de transformación republicana de Australia fue rechazada.

El Comité Asesor fue un órgano similar al que se constituiría en Irlanda unos años después - Constitution Review Group (1996)—y su función consiste en asesorar al gobierno sobre un posible cambio constitucional. Concretamente, la propuesta formulada en Australia era el sometimiento a referéndum de la conversión de Australia en una República cuyo presidente fuera elegido por una mayoría de dos tercios, emitida por la Cámara de Representantes y el Senado, reunidos en sesión conjunta sobre un candidato propuesto por el gobierno. La llegada al gobierno de la Coalición liberal nacional de John Howard en $1996^{52}$, tras la derrota laborista, introdujo un modelo diferente en virtud del cual el cambio de la forma de gobierno tendría que ser debatida por una Convención Constitucional ${ }^{53}$. Su composición fue de 152 miembros, de los cuales 76 fueron delegados electos por los ciudadanos en un complejo proceso de voto por correo, sin precedentes en el país austral, sobre la base de la representación parlamentaria de los distintos estados de la federación, mientras que los restantes 76 delegados fueron elegidos por el gobierno federal. De estos, 40 fueron delegados parlamentarios de los parlamentos federal, de los Estados y de los Territorios. Los restantes 36 miembros fueron delegados de carácter no parlamentario designados con la finalidad de conseguir una representación de todos los sectores, aborígenes, representantes nativos de las Islas Torres Strait, representantes de los gobiernos locales, de la juventud y de las mujeres ${ }^{54}$. La conclusión del trabajo de esta Convención Constitucional fue adoptar un modelo de elección presidencial indirecta. Un Presidente elegido por una mayoría de 2/3, en sesión conjunta de ambas cámaras, sobre una propuesta conjunta del primer ministro y del líder de la oposición y que podría ser depuesto por el primero. Previsiblemente, este elitismo ${ }^{55}$ subyacente en la designación y remoción fue lo que condujo a la derrota de la propuesta en el referéndum de 6 de noviembre de $1999^{56}$.

Un segundo ejemplo de Convención cuasi-constitucional nos lleva al proceso de elaboración de la Carta de los Derechos Fundamentales de la Unión Europea que los Presi-

51 ODOWD, T. J., «In the Constitutional Hall of Mirrors: Reflections of the Irish Constitution from abroad». Ponencia presentada a la tercera conferencia anual de Irish Society of Comparative Law, 30 de abril de 2011. (http://papers.ssrn.com/sol3/papers.cfm?abstract_id=1938692).

52 Véae: http://www.aec.gov.au/Elections/federal_elections/1996/index.htm

53 La recopilación de todos los archivos referidos a la realización de dicha Convención Constitucional puede hallarse en: THE AUSTRALIAN ELECTORAL COMMISSION’S, 1997 Consitutional Convention Elections Report and Statistics, http://www.aec.gov.au/elections/constitutional_convention/index.htm.

54 En una elección celebrada entre noviembre y diciembre de 1997, donde tras la inscripción como elector, en este caso no obligatoria como ocurre con las restantes elecciones en Australia, se procedió a seleccionar a los candidatos. Éstos se postulaban, previo pago de una tasa no rembolsable de 500 \$ por candidato. La votación fue por correo. Se emitieron 12 millones de paquetes postales, de los que retornaron la mitad, con lo que fue una elección escasamente ecológica en la que se usaron 160 toneladas de papel. El Estado con más electos fue Nueva Gales del Sur (20) y los que menos, la capital y los Territorios del Norte con 2 respectivamente. http://www.aec.gov.au/elections/constitutional_convention/index.htm.

55 O`DOWD, T. J., «In the Constitutional Hall of Mirrors: Reflections of the Irish Constitution from abroad». op. cit., pág. 6.

56 Cfr. http://www.aec.gov.au/elections/referendums/1999_Referendum_Reports_Statistics/1999.htm 
dentes del Parlamento Europeo, del Consejo y de la Comisión firmaron y proclamaron el 7 de diciembre de 2000 con ocasión del Consejo Europeo de Niza. Ésta fue encomendada inicialmente a una «Convención» en virtud de un mandato acordado por el Consejo Europeo de Colonia, celebrado el 3 y 4 de junio de $1999^{57}$.

«El Consejo Europeo considera que un proyecto de Carta de derechos fundamentales de la Unión Europea debe ser redactado por un órgano en el que participen delegados de los Jefes de Estado y de Gobierno y del Presidente de la Comisión Europea y miembros del Parlamento Europeo y de los Parlamentos nacionales. Deberían participar como observadores representantes del Tribunal de Justicia Europeo y habría de escucharse la opinión de representantes del Comité Económico y Social, del Comité de las Regiones, de grupos sociales y de expertos. Las funciones de secretaría correrán a cargo de la Secretaría General del Consejo». (Anexo IV. Decisión del Consejo Europeo relativa a la elaboración de una Carta de Derechos Fundamentales de la Unión Europea).

La Convención se constituyó en diciembre de 1999 y aprobó el proyecto el 2 de octubre del año 2000. La Composición efectiva de esa Convención se circunscribe a los siguientes miembros: 15 representantes de los Jefes de Estado y de Gobierno; 30 representantes de los Parlamentos nacionales; 16 representantes del Parlamento europeo y 1 representante de la Comisión. La Convención estuvo presidida por Roman Herzog, anterior presidente de la República Federal Alemana y del Tribunal Constitucional Alemán. A esta composición hay que añadir la presencia de observadores procedentes del Tribunal de Justicia de las Comunidades Europeas, del Comité de las Regiones, del Comité Económico y Social, del Defensor del Pueblo Europeo y del Consejo de Europa ${ }^{58}$. La participación ciudadana se canalizó a través de las contribuciones formuladas por asociaciones, especialmente de carácter no gubernamental ${ }^{59}$.

Finalmente, hemos de aludir a dos ejemplos desarrollados en la década pasada de Convenciones Constitucionales, designadas como Asambleas de Ciudadanos, habilitadas con el objeto de proponer la reforma de los sistemas electorales de dos provincias canadienses, la Columbia Británica y Ontario ${ }^{60}$. Ambas propuestas fueron rechazadas en sendos referenda ${ }^{61}$. Para formar su composición se acudió a la designación aleatoria de ciudadanos, un total de 160, a los que se les sometió a un proceso de aprendizaje en materia electoral, con lo que la contaminación por parte de los expertos pudo influenciar en un alejamiento de su propuesta a la defendida por los respectivos gobiernos liberales de cada provincia, lo que conduciría a su rechazo final.

57 JIMENA QUESADA, L. y ALEGRE MARTÍNEZ, M.A, Fundamentos constitucionales de la Unión Europea, Madrid, Biblioteca Nueva, 2006, págs. 115-157.

58 http://www.europarl.europa.eu/charter/composition_en.htm

59 http://www.europarl.europa.eu/charter/civil/civilo_en.htm

60 Citizen's Asembly on Electoral Reform (British Columbia) (http://www.citizensassembly.bc.ca.public). Ontario citizens'Asembly on electoral reform (http://www.citizensassembly.gov.on.ca).

61 El trabajo de estas Asambleas ha sido tomado en consideración por el parlamento irlandés a los efectos de reformar el art. 16 de su Constitución, relativo al sistema electoral. Joint Committe on the Constitution fourth Report: Article16 of the Constitution - Review of the Electoral System for the election of members to Dáil Éireann, Final Report (Julio 2010), pág. 161. (http://www.oireachtas.ie/documents/committees30thdail/j-constitution/report_2008/20100722.pdf). 


\section{BASES PARA LA CREACiÓN DE UNA CONVENCIÓN CONSTITUCIONAL EN IRLANDA}

Con el objetivo de articular un proceso en virtud del cual asegurar que la «Constitución irlandesa afronte los retos del siglo XXI» ${ }^{62}$, el gobierno resultante de las elecciones celebradas en Irlanda el 25 de febrero de 2011, integrado por la coalición del Fine Gael y los laboristas ${ }^{63}$, acordó en su programa de gobierno afrontar el proceso de reforma constitucional sobre una serie de materias abanderadas por los distintos colores, verbigratia el Fine Gael abrazó la supresión del Senado; para el derrotado Fianna Fail la prioridad era la reforma del sistema electoral. Finalmente, la coalición gobernante incluyó en el programa de gobierno, cinco cuestiones prioritarias a las que nos hemos referido en el epígrafe precedente, y se comprometió a establecer una Convención Constitucional aceptando para ello una propuesta formulada desde la oposición por los Laboristas y por el Fianna Fáil_— ${ }^{64}$ a los efectos de tomar en consideración una «reforma constitucional comprehensiva» ${ }^{65}$. La primera advertencia es que estamos ante un proceso abierto, sincrónico a la redacción de este trabajo, por lo que en el mismo analizaremos el iter completado hasta el momento actual y las principales consecuencias del mismo.

El balance de esa propuesta, dieciséis meses más tarde de su formulación, se condensa en los siguientes asertos.

Aproximación material. Lo que se considera por los irlandeses como «un espacio en el que reflejar los valores y principios que definen quienes somos y adonde queremos dirigir nuestro Estado» ${ }^{66}$ se ha configurado hasta el momento en base a tres referentes sustantivos que deberán culminar en una resolución de ambas cámaras del Parlamento (Oireachtas). El programa de gobierno de 6 de marzo de 2011, la propuesta del gobierno para la creación de una Convención Constitucional, de 28 de febrero de $2012^{67}$ y, por el momento, la respuesta del gobierno a las alegaciones de la oposición sobre la propuesta para establecer una Convención Constitucional ${ }^{68}$.

La fijación del ámbito material sobre el que trabajará dicha Convención Constitucional se ha afrontado desde una perspectiva de delimitación positiva y negativa. El gobierno ha mantenido intangible la relación de cuestiones a abordar por parte de dicha Convención. Así, desde el programa de gobierno de 2011, estas cuestiones se concretan en las siguientes: revisión del sistema electoral parlamentario; reducir el mandato presidencial de 7 a 5 años y vincular esa elección a la celebración de las elecciones locales y al Parlamento Europeo; matrimonio entre personas del mismo sexo; reformar el artículo 41

62 Programme for Government 2011 (6 de marzo de 2011). P. 17. http://www.taoiseach.gov.ie/eng/Publications/Publications_2011/Programme_for_Government_2011.pdf

63 Para ver los resultados: http://electionsireland.org/results/general/31dail.cfm.

64 Aunque en 2009 fue el Fine Gael quien lanzó la propueta, en la campaña electoral serían los laboristas quienes la incorporaron en su programa electoral. McGEE, H., «Convention to discuss reform of Constitution», en Irish times, 6 de junio de 2012.

65 Programme for Government 2011 (6 de marzo de 2011). P. 17. http://www.taoiseach.gov.ie/eng/Publications/Publications_2011/Programme_for_Government_2011.pdf

66 O’GORMAN, C., «Constitutional Convention», en Irish Times, 11 de junio de 2012.

67 http://www.merrionstreet.ie/index.php/2012/02/constitutional-convention-government-proposals28-february-2012/.

68 http://www.merrionstreet.ie/index.php/2012/06/government-response-to-opposition-views-on-theproposal-to-establish-a-constitutional-convention/?cat $=$ 
de la Constitución en el que se contempla el trabajo de las mujeres en el hogar, a la par que potenciar la participación de las mujeres en la vida pública; suprimir la blasfemia de la Constitución y posible reducción de la edad mínima para votar de 18 a 17 años. Como colofón a esa agenda, positivamente acotada, se establece la posibilidad de que la Convención Constitucional realice recomendaciones sobre otras cuestiones que se considere necesario reformar en la Constitución, que siempre deberán posponerse al estudio de las citadas. El tratamiento de las mismas se estructura, marcando como primeras cuestiones a abordar la reducción del mandato presidencial a cinco años y el adelanto de la edad que permite votar a los 17 años. Sobre estas dos cuestiones la Convención deberá pronunciarse en el plazo de dos meses desde su constitución ${ }^{69}$. Se comprende que relegar tan ambicioso proyecto a cuestiones que se pueden calificar como «relativamente inconsecuentes» ${ }^{70}$ proyecte demasiadas sombras sobre dicha Convención. En un contexto de crisis, en el que en muchas ocasiones los ciudadanos parecen saber con más certeza cuáles son los fallos y qué rumbo tomar, ese encorsetamiento temático augura no demasiada popularidad de la Convención.

La delimitación negativa se centra sobre aquellas cuestiones sobre las que existe un compromiso de sometimiento a referéndum, como por ejemplo la supresión del Senado o los derechos de los niños.

Aproximación formal. El resultado del trabajo de la Convención Constitucional debe plasmarse en una Recomendación trasladada al Gobierno, que tendrá la obligación de responder en sede parlamentaria en un plazo máximo de cuatro meses. Como consecuencia del debate parlamentario, en el caso de que el gobierno adopte esa recomendación de reforma constitucional, algo facultativo, procederá a la convocatoria de referéndum. En consecuencia, la eficacia inmediata de tal Convención se ve muy mermada, más allá de la lealtad política con el instrumento de la Convención Constitucional. Si nos ceñimos al momento presente es bastante improbable que alguna de las Recomendaciones de dicha Convención constitucional se conviertan en una ley de reforma constitucional bajo el mandato del actual gobierno, especialmente cansado de batallar con dicho referéndum con el desgaste político que conlleva, así la derrota en el referéndum para otorgar más poder a los Comités del Parlamento (27 de octubre de 2011) y el absentismo del celebrado el 31 de mayo de 2012, sobre la ratificación del Tratado Fiscal.

\section{ESTRUCTURA Y FUNCIONES}

La descripción de los principales aspectos procesales que determinarán el funcionamiento de dicha Convención Constitucional los podemos reconducir a cuatro referentes: composición, método de trabajo, estructura organizativa y financiación.

La composición de la Convención se adelantó en la propuesta de 28 de febrero de 2012. Se conformará por 100 miembros, incluyendo un presidente que será fruto del consenso sobre alguien que reúna los requisitos de poseer una «excepcional habilidad y

69 Propuesta gubernamental de Convención Constitucional de 28 de febrero de 2012.

70 En ese sentido, O’MAHONEY, C., «This so-called Constitutional Convention is a charade», Irish Times, 7 de junio de 2012 . 
un alto grado de aceptación pública». 66 serán ciudadanos ordinarios. Los restantes 33 serán miembros del parlamento, así como un parlamentario de cada uno de los partidos políticos de Irlanda del Norte que acepten la invitación a formar parte de la Convención. Su designación se hará sobre la base de criterios de representatividad parlamentaria. Respecto a los ciudadanos el cometido de su designación — como contraste de la complejidad con que se afrontó ese proceso en Australia - recaerá en una empresa privada mediante una selección aleatoria a partir de los datos contenidos en el registro electoral. Se buscará un reparto paritario entre sexos y la mayor representación demográfica, geográfica y social posible $e^{71}$. El interrogante aquí radica en la «voluntariedad» con la que se concibe la presencia en dicha Convención, o en otros términos la obligatoriedad de la misma. Esta circunstancia es decisiva porque condicionará el interés de los participantes y, en consecuencia, los resultados a alcanzar.

Con respecto a la presencia de diferentes colectivos, ciudadanos de Irlanda del Norte, irlandeses residentes en el extranjero, Grupos de Interés o Expertos, el gobierno se ha inclinado por la presencia de ciudadanos, sin ninguna adscripción, abiertos, eso sí a la recepción de propuestas de estos colectivos, especialmente a través de las nuevas tecno$\log$ ías $^{72}$.

El trabajo de la Convención podrá estructurarse en secciones que aborden cuestiones separadamente, pero se exige que su reunión sea plenaria para aprobar una Recomendación. A parte de esa mínima base, se remite a la propia potestad autoorganizativa de la Convención el ultimar los detalles de sus pautas de trabajo.

El análisis de la estructura del mismo nos hace reparar en tres cuestiones: la organización administrativa sobre la que se asienta, la presencia de expertos y la apertura de un período de formación para sus componentes. El contexto de crisis en el que se erige obliga a que su dotación administrativa se haga en base a los efectivos existentes, considerándose por el gobierno en la respuesta formulada a la oposición la eventual contratación de jóvenes desempleados para dicha tarea.

La presencia de expertos se ha excluido de la Convención, pero no se ha renunciado a su asesoramiento. El colectivo designado para ello serán politólogos, constitucionalistas y académicos. El riesgo de aleccionamiento de los miembros de la Convención se trata de eludir con esa opción, se rehúye del tecnicismo en aras del populismo a priori más neutral.

Vinculado a ello, la formación de los miembros de la convención será irrenunciable cuando aborden tareas específicas que requieran unos mínimos conocimientos para recomendar con rigor una reforma constitucional. El temor a los «impressive professors» del ejemplo australiano ha presentado esta fase como una de las más frágiles del proceso.

Finalmente, la cuestión de la financiación se resuelve con una dotación de 300.000 $€$, para el ejercicio 2012. Resulta curioso verificar la procedencia de esa suma, el Departamento del Presidente (Taoiseach).

71 Entrevista a HARVEY, C., School of Law, Queen's University, Belfast: « What kind of Constitutional Convention do you want?», The journal. ie, 23 de abril de 2012, http://www.thejournal.ie/readme/columnwhat-kind-of-constitutional-convention-do-you-want/

72 Debates parlamentarios, Dáil Éireann Debate, Vol. 768, n.1 1, 12 de junio de 2012. (http://debates.oireachtas.ie/dail/2012/06/12/00100.asp). 


\section{APUNTES DESDE EL CONSTITUCIONALISMO IRLANDÉS}

La máxima de "Ireland being used as a cautionary example» ${ }^{73}$ ha de seguirse fielmente en esta última reflexión que sólo trata de enlazar con las cascadas de sensaciones que este contexto de crisis está generando, especialmente para los constitucionalistas.

En primer lugar, cualquier respuesta que el orden constitucional ofrezca debe vencer la resistencia a la tiranía de lo sincrónico. La vocación de proyección temporal de la Constitución debe obviar petrificar compromisos bienales, trienales o quinquenales y optar por fórmulas operativas en la realidad inmediata, pero válidas en supuestos de hecho diferentes.

En segundo lugar, apostar por la idea de que un sistema estable, constitucional y económicamente sólido, debe apostar por vincular a las élites y a las masas en un proyecto compartido. Cuando el progreso no tiene una proyección general, cuando el Estado de bienestar construido no se extiende a toda la sociedad, el riesgo de fragmentación es elevado y se multiplica en el momento en el que las circunstancia económicas empeoran. A ese respecto hemos de asumir una de las principales lecciones extraídas de la crisis: no afrontar los problemas macroeconómicos significa años de oportunidades perdidas. La apuesta por una economía inteligente en la que se cree riqueza en el mundo real y no en el de la burbuja es el primer reto.

Una tercera reflexión es que debemos reorientar nuestro sistema jurídico y político hacia un modelo más robusto y deliberativo. La sensación que hemos albergado durante la crisis acerca de que los ciudadanos de a pie sabemos mejor o creemos saber con más claridad lo que hay que hacer que nuestros políticos es un indicador de una debilidad seria de nuestro sistema. Es el momento de renovación de la clase política, apostar por una tecnificación, una cualificación, sobre la que proyectar una ideología y una orientación política, pero traducible en políticos altamente formados, con experiencia profesional consolidada en diferentes ámbitos laborales. El período del oficio de político ha de cerrarse. Tendríamos que evolucionar del político por vocación y profesión al político por necesidad. Es decir la necesidad de políticos cualificados, siendo necesario proyectar su experiencia profesional más allá del ámbito donde la han gestado y proceder a cubrir esa necesidad social de personas expertas a la hora de decidir y guiar la trayectoria del Estado ${ }^{74}$.

Un cuarto apunte, se centra en la esencialidad o no de las reformas constitucionales. De la cotidianeidad con que en Irlanda se concibe ese proceso al temor, demostrado infundado a la luz de la reciente reforma de nuestra Constitución, de afrontar su reforma es preciso hallar un punto medio que asuma la normalidad de la reforma con la necesidad de que su implementación sea necesaria y participada. El aislamiento de los políticos de la sociedad, parecen dos líneas paralelas destinadas a no cruzarse jamás, exige como primer referente un consenso entre las fuerzas políticas. En lo esencial, organización territorial,

73 O'DOWD, T. J., «In the Constitutional Hall of Mirrors: Reflections of the Irish Constitution from abroad». Op. cit., pág. 3.

74 La sugerentes reflexiones del profesor Alzaga completan las ideas que a este respecto se formulan. Cfr. ALZAGA, O., «Hablando con Óscar Alzaga Villaamil», en Cuadernos de la Tarde. Revista del Gabinete de Calidad de Vida de la Universidad de Granada, n. ${ }^{\circ} 7$, junio 2012, págs. 22-27. 
Unión Europea, Constitución económica, no puede haber disenso. La propuesta de una convención constitucional puede resultar atractiva si su creación sirviese al menos para reactivar y desentumecer la vía parlamentaria que parece anquilosada en la cultura de los desencuentros y la oposición por sistema.

Un quinto apunte, es una apelación a la necesidad de priorizar el papel de la Constitución económica y de los debates presupuestarios. Un mayor rigor en los mismos, un mayor esfuerzo en su trasparencia. Los constitucionalistas hemos aprendido en esta crisis que un control del gasto público reposa sobre el corazón del control parlamentario sobre el gobierno.

Un sexto apunte, es una apelación a los principios de eficiencia y responsabilidad en el ámbito institucional y en la organización del Sector Público. La responsabilidad de los gestores debe ir acompañada de una responsabilidad de los usuarios, la apelación a la transparencia es irrenunciable en esta cuestión. No duplicidades, no asesoramientos externos cuando hay cuerpos cualificados que hacen ese trabajo en el seno de las instituciones, y más responsabilidad de los políticos, a nivel político y jurídico.

Un apunte final es una reflexión a la capacidad de aprendizaje. Hasta ahora nuestros gobernantes han manifestado una débil habilidad para aprender de errores pasados y adelantar futuras necesidades. La política y la gestión adaptativa y creativa ha sido inexistente. Evitar lealtades que derivan de endogamias peligrosas (política y sector bancario), limitar la politización de los agentes financieros y de quien los controla y concienciarnos de la importancia de realizar una supervisión del gasto público como un todo en relación a los recursos. La apuesta por la participación social, incluso a nivel de reforma constitucional, puede ayudar a identificar los aspectos más vulnerables, lejos de monopolios inmobiliarios o problemas creados y alimentados mediáticamente, y recentrar el sistema hacia las preocupaciones de la sociedad, las reales no las aisladas en burbujas.

$$
* * *
$$

TITLE: Constitutional Convention versus Constitutional Amendment: Analysis over Irish Déjà vu from the point of view of Spanish Constitutional reform.

AвSTRACT: This article focusses on Irish answers to the economic crisis. The full force of the sovereign debt crisis has been affected Ireland from 2008. The failures in the policy-making and regulatory systems have caused a critical eye on traditional ways of doing business in the political system and the public service. The paths that Ireland has followed are two. First, the constitutional amendment mechanism. It pays attention to constitutional precepts that ruler this constitutional reform, mainly articles 46 and 47. The referendum has developed a key element in this system. Ireland has developed 23 constitutional reforms nowadays. The second important element is a Constitutional Convention. This body will allow a group of randomly selected citizens to deliberate and make recommendations upon a number of areas of political reform. Many of the changes envisaged offers an space for reflection about the viability of a macro political and constitutional change strategy that the bailout require.

RESUMEN: El presente artículo se centra en el estudio de la gestación de la crisis económica en Irlanda y en el análisis de las respuestas constitucionales a la misma. En primer lugar se analiza el papel de la reforma constitucional, estudiando el mecanismo diseñado en los arts. 46 y 47 de la Constitución. El carácter preceptivo del referéndum ha reforzado la implicación social en un total de 23 reformas aprobadas hasta la fecha. De especial trascendencia, como reacciones constitucionales ante el contexto de crisis, podemos destacar la reforma de 2011 relativa a la reducción del salario de los jueces y la afrontada en 2012, como consecuencia de la ratificación del Tratado de Estabilidad. Un segundo contenido de este trabajo expone una «relativa» novedad en el panorama constitucional, la de la Convención Constitucional. Concebida como un órgano deliberativo y participativo, estamos ante un mecanismo que trata de paliar una común demanda de las democracias 
actuales de fomentar la participación social como complemento a las limitaciones de los cauces parlamentarios tradicionales, subyugados a demasiadas lealtades, partidistas, endogámicas que impiden el ejercicio de sus funcione de canalización de la voluntad popular en el seguimiento de los asuntos públicos.

KEY WORDS: Constitutional amendments. Constitutional convention.

Palabras Clave: Crisis económica. Reforma constitucional. Convención constitucional.

FECHA DE RECEPCIÓN: 6.06.2012. FECHA DE ACEPTACIÓN: 19.07.2012 